\title{
The Burden of Obstructive Lung Disease (BOLD) study in Australia
}

\author{
The implications for practice and policy of new national prevalence data
}

Christine F McDonald MB BS(Hons), PhD, FRACP Respiratory Physician

Nicholas Glasgow MB ChB, MD, FRACGP Dean of Medical School

1 Department of Respiratory and Sleep Medicine, Austin Hospital ustin Hospita

Melbourne, VIC

2 College of Medicine Biology and Environment

Australian Nationa University, Canberra, ACT.

christine.mcdonald@ austin.org.au

doi: 10.5694/mjal3.1003

Research p 144 $\mathrm{n}$ this issue of the Journal, Toelle and colleagues provide up-to-date information about obstructive lung diseases in Australia in their report on the Australian arm of the Burden of Obstructive Lung Disease (BOLD) study. ${ }^{1}$ The BOLD study is an international collaboration comparing rates of obstructive lung disease in people aged 40 years or older in 12 countries. Sydney's place in this international league table was reported previously, ${ }^{2}$ but now we have access to Australia-wide data, with six sites chosen specifically to ensure adequate representation of the states and territories and, importantly, to include Indigenous groups. Given that the overall participation rate in this study was only $27 \%$, it is possible that the disease burden may be greater or less than estimated, but these data nevertheless allow more robust international comparisons than have previously been possible.

The overall prevalence estimate of moderate to very severe chronic obstructive pulmonary disease (COPD) classified on spirometry as Global Initiative for Chronic Obstructive Lung Disease (GOLD) ${ }^{3}$ Stage II or higher was $7.5 \%$. Among men and women, the estimates were $6.9 \%$ and $8.1 \%$, respectively, representing the lower end of the international range for men and the middle for women. Although still commonly thought of as a disease of older men who smoke, COPD rates in women are now overtaking those in men, as smoking rates in Australian women did not peak until the late 1970s while rates in men have fallen progressively since the end of World War II. In this sample of older adults, $50 \%$ of participants had smoked cigarettes at some time in their lives, highlighting the need for continued efforts to reduce tobacco smoking rates.

In addition to giving greater precision to the prevalence estimates for COPD in Australia, this study provides a timely baseline against which the future impact on lung health of the Australian Government's world-leading tobacco plain-packaging legislation and associated comprehensive suite of preventive measures ${ }^{4}$ can be assessed.

The study also points to the complex interaction between asthma and COPD and the essential role of spirometry in diagnosing these conditions. It may be argued that the degree of bronchodilator reversibility used in the BOLD methodology to suggest an asthma diagnosis can also be seen in some patients with known COPD who have no history or associated features suggestive of asthma. However, it is certainly true that a spirometric diagnosis of airflow obstruction, or its absence, is key when a practitioner is faced with a patient who complains of chronic respiratory symptoms, which might be due to asthma, COPD, heart disease, obesity, a combination of these, or other causes. It is clinically important to know whether a person has asthma, COPD or both, as the management approach differs, and drug choices and doses vary. In 2011, an Australian study of asthma and COPD in general practice patients found that, of 2869 respondents, $8.5 \%$ had diagnosed asthma without COPD, 3.1\% had COPD without asthma, and 2.2\% had both COPD and asthma. ${ }^{5}$ Yet the validity of the diagnoses and the generalisability of these data are questionable, given that general practitioners prefer to diagnose COPD on clinical grounds. ${ }^{6}$ In a recent study in primary care, more than $40 \%$ of patients labelled as having COPD clinically had the diagnosis refuted when they underwent spirometry. ${ }^{7}$ These findings have important implications, including the unnecessary risk of adverse effects from treatment for a condition the patient does not have, the possibility that the true cause of the patient's respiratory symptoms may not be identified and treated, and the cost implications for the patient and the health system.

Underdiagnosis of COPD is placed in stark relief when considering patients at the other end of the health care spectrum - those admitted to hospital. A study of patients undergoing spirometry while hospitalised for a nonCOPD condition, who were unaware of having obstructive lung disease, found that $16 \%$ had evident airflow obstruction. ${ }^{8}$ Even in patients admitted for the first time with a primary diagnosis of "acute exacerbation of COPD", lack of a previous diagnosis of COPD is high (34\%), despite the severity of the disease. ${ }^{9}$ Using spirometry to ensure earlier diagnosis is crucial to prevent such episodes, which are associated with a concerning 5 -year mortality rate of $50 \%{ }^{10}$

Optimising management for any chronic condition assumes an accurate diagnosis has been made. Continuing efforts are required to ensure that spirometric assessments undertaken in community settings are providing valid results.

Perhaps the biggest challenge in managing COPD, as with most chronic conditions, is how the health system can be better engineered to provide patient-centred services to people with multimorbidity. The current system has been built around single diseases. This is reflected in the way research is conducted, guidelines are developed, Medicare item numbers are structured and, particularly in 
hospital settings, how health care services are configured. Most people over the age of 50 years presenting with a chronic disease have multiple coexisting diseases. They need a system that supports them to achieve balance in and between their lives and their illnesses. ${ }^{11,12}$

Local hospital networks and Medicare Locals offer new platforms for innovative approaches to treating people with chronic conditions. Could these entities identify common clusters of multimorbidity in their area and, using existing guidelines for each condition in the cluster, develop and promote a "synthesis guideline" outlining optimal practice? Or, for a COPD-specific need, could they look at spirometry services available in their area and develop and promote innovative approaches that increase the availability of easily accessible spirometry, as has been done in the Netherlands? ${ }^{13}$

Toelle and colleagues do indeed provide us with a "BOLD" reminder of the burden of COPD in Australia. Bold actions are required in clinical practice, health service configuration and health policy to meet the challenges this disease presents. The Australian Government's tobacco plain-packaging legislation is a great start.

Competing interests: No relevant disclosures.

Provenance: Commissioned; externally peer reviewed.

1 Toelle BG, Xuan W, Bird TE, et al. Respiratory symptoms and illness in older Australians: the Burden of Obstructive Lung Disease (BOLD) study. Med J Aust 2013; 198: 144-148.
2 Buist AS, McBurnie MA, Vollmer WM, et al; BOLDCollaborative Research Group. International variation in the prevalence of COPD (the BOLD Study): a population-based prevalence study. Lancet 2007; 370: 741-750.

3 Global Initiative for Chronic Obstructive Lung Disease. Global strategy for the diagnosis, management and prevention of chronic obstructive pulmonary disease. Updated 2010. http://www.goldcopd.org/Guidelines/guideline-2010gold-report.html (accessed Jan 2013).

4 Australian Government Department of Health and Ageing. Plain packaging of tobacco products. http://www.health.gov.au/internet/main/publishing.nsf/ content/tobacco-plain (accessed Jan 2013).

5 Family Medicine Research Centre, University of Sydney. SAND abstract No. 182 from the BEACH program: chronic obstructive pulmonary disease (COPD) in general practice patients (2). Sydney: FMRC, 2012. http://sydney.edu.au/ medicine/fmrc/publications/sand-abstracts/182-COPD.pdf (accessed Jan 2013).

6 Walters JA, Walters EH, Nelson M, et al. Factors associated with misdiagnosis of COPD in primary care. Prim Care Respir J 2011; 20: 396-402.

7 Zwar NA, Marks GB, Hermiz O, et al. Predictors of accuracy of diagnosis of chronic obstructive pulmonary disease in general practice. Med J Aust 2011; 195 168-171.

8 Yamasaki A, Hashimoto K, Hasegawa Y, et al. COPD is frequent in conditions of comorbidity in patients treated with various diseases in a university hospital. Int J Chron Obstruct Pulmon Dis 2010; 5: 351-355.

9 Bastin AJ, Starling L, Ahmed R, et al. High prevalence of undiagnosed and severe chronic obstructive pulmonary disease at first hospital admission with acute exacerbation. Chron Respir Dis 2010; 7: 91-97.

10 Garcia-Aymerich J, Serra Pons I, Mannino DM, et al. Lung function impairment, COPD hospitalisations and subsequent mortality. Thorax 2011; 66: 585-590.

11 Jeon YH, Jowsey T, Yen L, et al. Achieving a balanced life in the face of chronic illness. Aust JPrim Health 2010; 16: 66-74.

12 Jowsey T, Jeon YH, Dugdale P, et al. Challenges for co-morbid chronic illness care and policy in Australia: a qualitative study. Aust New Zealand Health Policy 2009; 6: 22

13 Tsiligianni IG, van der Molen T, Tzanakis NE, et al. A telehealth integrated asthma-COPD service for primary care: a proposal for a pilot feasibility study in Crete, Greece. BMC Res Notes 2010; 3: 198. doi: 10.1186/1756-0500-3-198. 